\title{
BEST COAPPROXIMATION IN METRIC LINEAR SPACES
}

\author{
T. D. NARANG AND S. P. SINGH
}

\begin{abstract}
In order to obtain some characterizations of real Hilbert spaces among real Banach spaces, a new kind of approximation, called best coapproximation, was introduced in normed linear spaces by $C$. Franchetti and M. Furi [3] in 1972. Subsequently, the study was pursued in normed linear spaces and Hilbert spaces by H. Berens, L. Hetzelt, T. D. Narang, P. L. Papini, Geetha $S$. Rao and her students, Ivan Singer and a few others (see, e.g., [1], [4], [7], [9], [13 to 15], and [ 17 to 20$]$ ). In this paper, we discuss best coapproximation in metric linear spaces thereby generalizing some of the results proved in [3], [7], [13], and [18]. The problems considered are those of existence of elements of best coapproximation and their characterization, characterizations of coproximinal, co-semi-Chebyshev and co-Chebyshev subspaces, and some properties of the best coapproximation map in metric linears spaces.
\end{abstract}

\section{Introduction}

The main object of the theory of best approximation is to seek a solution to the problem: Given a subset $G$ of a metric space $(X, d)$ and an element $x \in X$, find an element $g_{0} \in G$ such that

$$
d\left(x, g_{0}\right) \leq d(x, g) \text { for all } g \in G
$$

The set of all such $g_{0} \in G$ (if any), called the set of best approximation of $x$ by elememts of $G$, is denoted by $P_{G}(x)$. Clearly

$$
P_{G}(x)=\left[\cap_{g \in G} B(x, d(x, g))\right] \cap G,
$$

where $B(x, d(x, g))$ denotes the closed ball in $X$ with centre $x$ and radius $d(x, g)$.

As a counterpart to best approximation, another kind of approximation, called best coapproximation, was introduced by Franchetti and Furi [3], who considered those elements $g_{0} \in G$ satisfying

$$
d\left(g_{0}, g\right) \leq d(x, g) \text { for all } g \in G
$$

The set of all such $g_{0} \in G$ (if any) is denoted by $R_{G}(x)$. Clearly

$$
R_{G}(x)=\left[\cap_{g \in G} B(g, d(x, g))\right] \cap G .
$$

Received October 3, 1995; revised September 8, 1998.

1991 Mathematics Subject Classification. 45H15.

Key words and phrases. Best coapproximation, coproximinal co-semi-Chebyshev, co-Chebyshev, best coapproximation map. 
An element $g_{0} \in G$ satisfying (1) is called a best approximation to $x$ in $G$, and satisfying (2) is called a best coapproximation to $x$ in $G$. The set $G$ is said to be proximinal (respectively, coproximinal) if $P_{G}(x)$ (respectively, $R_{G}(x)$ ) is non empty for each $x$ in $X$. It is said to be semi-Chebyshev (respectively, co-semi-Chebyshev) if $P_{G}(x)$ (respectively, $R_{G}(x)$ ) contains at most one element for each $x$ in $X$ and it is said to be Chebyshev (respectively, co-Chebyshev) if $P_{G}(x)$ (respectively, $R_{G}(x)$ ) contains exactly one element for each $x$ in $X$. If $D\left(P_{G}\right)=\left\{x \in X: P_{G}(x) \neq \emptyset\right\}$ (respectively, $D\left(R_{G}\right)=\left\{x \in X: R_{G}(x) \neq \phi\right\}$ ), the mapping $P_{G}: D\left(P_{G}\right) \rightarrow G$ (respectively, the mapping $R_{G}: D\left(R_{G}\right) \rightarrow G$ ), defined by $x \rightarrow P_{G}(x)$ (respectively, $x \rightarrow R_{G}(x)$ ) is called the best approximation map or metric projection (respectively, best coapproximation map or metric coprojection). In general, $D\left(P_{G}\right)$ (respectively, $\left.D\left(R_{G}\right)\right) \neq X$ and the mapping $P_{G}$ (respectively, $R_{G}$ ) is multivalued on $D\left(P_{G}\right) \mid G$ (respectively, $D\left(R_{G}\right) \mid G$ ), but the restriction of the mapping $P_{G}$ (respectively, $R_{G}$ ) to $G$ is single-valued. We have $D\left(P_{G}\right)$ (respectively, $\left.D\left(R_{G}\right)\right)=X$ if $G$ is proximinal (respectively, coproximinal) and is single-valued on $X$ if $G$ is Chebyshev (respectively, co-Chebyshev).

As in the case of best approximation, the theory of best coapproximation has been developed to a large extent in normed linear spaces and in Hilbert spaces by H. Berens and U. Westphal [1], C. Franchetti and M. Furi [3], L. Hetzelt [4], T. D. Narang [7], [9], P. L. Papini and I. Singer [13], Geetha S. Rao and her students [4], [15], [17], [18], U. Westphal [20] and a few others. Geetha S. Rao was the first to develop the theory of best coapproximation after the appearance of the paper of Papini and Singer [13]. In a series of papers she and her students have proved many results on best coapproximation in normed linear spaces. The situation in the case of metric linear spaces is somewhat different. Whereas many successful attempts have been made to develop the theory of best approximation in metric linear spaces (although the theory is comparatively less developed than that in normed linear spaces due to the non-convexity of spheres, lack of duality theory in metric linear spaces, etc.), the theory of best coapproximation in such spaces is yet to make a beginning. The present paper is a step in this direction. We discuss in this paper some results on existence of elements of best coapproximation and their characterization, characterizations of coproximinal, co-semi-Chebyshev and coChebyshev subspaces and some properties of the best coapproximation map in metric linear spaces.

\section{Existence and Characterizations of Elements of Best Coapproximation}

In this section, we discuss some results on the existence and characterization of elements of best coapproximation in metric linear spaces. We start with listing a few elementary observations.

Observation 2.1. If $G$ is a subset of a metric space $(X, d)$, then $G \subset R_{G}(x)$ whenever the diameter of $G$ is smaller than $\operatorname{dist}(x, G)$. 
Observation 2.2. If $G$ is a convex subset of a strongly locally convex metric linear space (a metric linear space in which all spheres are convex-see [6] $(X, d)$ then $R_{G}(x)$ is a convex set.

Observation 2.3. If $G$ is a linear subspace of a metric linear space $(X, d)$ and $R_{G}^{-1}(0)=\left\{x \in X: 0 \in R_{G}(x)\right\}$, then

(i) $R_{G}^{-1}(0)$ is a closed set containing 0 ,

(ii) $g_{0} \in R_{G}(x) \Leftrightarrow 0 \in R_{G}\left(x-g_{0}\right)$ i.e., $x-g_{0} \in R_{G}^{-1}(0)$, and

(iii) for $g \in G$, we have $z \in R_{G}^{-1}(0) \Leftrightarrow g \in R_{G}(g+z)$, i.e., $g+z \in R_{G}^{-1}(g)$.

The following theorem gives a necessary and sufficient condition under which $R_{G}(x) \neq$ $\emptyset$ :

Theorem 1. If $G$ is a linear subspace of a metric linear space $(X, d)$, then $R_{G}(x) \neq \emptyset$ for some $x \in X \backslash G$ if and only if $R_{G}^{-1}(0)$ is not a singleton.

Proof. By Observation 2.3 (i), $0 \in R_{G}^{-1}(0)$. Suppose, $g_{0} \in R_{G}(x)$ for some $x \in X \backslash G$. Then by Observation 2.3 (ii), $0 \neq x-g_{0} \in R_{G}^{-1}(0)$ and so $R_{G}^{-1}(0)$ is not a singleton.

Conversely, suppose $R_{G}^{-1}(0)$ is not a singleton. Then there exists an $x \neq \emptyset \in R_{G}^{-1}(0)$ and so $0 \in R_{G}(x)$ i.e., $R_{G}(x) \neq \emptyset$ for some $x \in X \backslash G$.

It may be remarked that a similar result holds for $P_{G}(x)$.

It was shown by Johnson [5] that if $(X, d)$ is a metric space and $x_{0}$ is a fixed point of $X$ then the set

$$
X_{0}^{\#}=\left\{f: X \rightarrow \mathbb{R} \sup _{\substack{x \neq y_{x, y} \in x\\}} \frac{|f(x)-f(y)|}{d(x, y)}<\infty, \quad f\left(x_{0}\right)=0\right\}
$$

with the usual operations of addition, and multiplication by real scalars, normed by

$$
\|f\|_{X}=\sup _{x \neq y_{x, y} \in x} \frac{|f(x)-f(y)|}{d(x, y)}, \quad f \in X_{0}^{\#}
$$

is a Banach space (even a conjugate Banach space). Using this idea of Johnson, we prove:

Theorem 2. If $G$ is a linear subspace of a metric linear space $(X, d), x \in X \mid G$ and $g_{0} \in G$ then $g_{0} \in R_{G}(x)$ if for every $g \in G$ there exists an $f^{g} \in X_{0}^{\#}$ with the following properties:

(i) $\left|f^{g}(x)-f^{g}(y)\right| \leq d(x, y)$ for all $x, y \in X$,

(ii) $f^{g}\left(x-g_{0}\right)=0$, and

(iii) $f^{g}\left(g_{0}-g\right)=d\left(g_{0}, g\right)$. 
Proof. Suppose for every $g \in G$ there exists an $f^{g} \in X_{0}^{\#}$ satisfying (i), (ii), and (iii). Consider

$$
\begin{aligned}
d(x, g) & \geq\left|f^{g}(x)-f^{g}(g)\right|, \text { by (i), } \\
& =\left|f^{g}\left(x-g_{0}+g_{0}-g\right)\right| \\
& =\left|f^{g}\left(g_{0}-g\right)\right|, \text { by (ii) } \\
& =d\left(g_{0}, g\right), \text { by (iii) }
\end{aligned}
$$

i.e., $d\left(g_{0}, g\right) \leq d(x, g)$ for all $g \in G$ and so $g_{0} \in R_{G}(x)$.

Problem 2.1. If $g_{0} \in R_{G}(x)$ then can we find an $f^{g} \in X_{0}^{\#}$ satisfying (i), (ii), and (iii)?

Remark 2.1. A result similar to Theorem 2 and its converse were given by $\mathrm{G}$. Pantelidis [12] for $P_{G}(x)$ in metric linear spaces and for $R_{G}(x)$ by Papini and Singer [13] in normed linear spaces.

An element $x$ of a metric linear space $(X, d)$ is said to be orthogonal to another element $y \in X$ (see [6]), and we write $x \perp y$, if $d(x, 0) \leq d(x, \alpha y)$ for every scalar $\alpha . x$ is said to be orthogonal to a subset $G$ of $X(x \perp G)$ if $x \perp y$ for all $y \in G$. This definition of orthogonality is similar to that given by G. Birkhoff [2]. It is known (see [6]) that if $G$ is a linear subspace of a metric linear space $(X, d), x \in X \mid \bar{G}$ and $g_{0} \in G$ then $g_{0} \in P_{G}(x)$ if and only if $x-g_{0} \perp G$. It was proved in [3] that if $G$ is a linear subspace of a normed linear space $X$ and $g_{0} \in G$ then $g_{0} \in R_{G}(x)$ if and only if $G \perp x-g_{0}$. In metric linear spaces, the following is easy to prove:

If $G$ is a linear subspace of a metric linear space $(X, d)$ and $g_{0} \in G$, then $g_{0} \in R_{G}(x)$ if $G \perp\left(x-g_{0}\right)$ or if $G-g_{0} \perp\left(x-g_{0}\right)$.

Problem 2.2. Is the converse also true, i.e., if $g_{0} \in R_{G}(x)$, then can we prove that. $G \perp\left(x-g_{0}\right)$ or $G-g_{0} \perp\left(x-g_{0}\right)$ ?

Using orthogonality in metric linear space, we have:

Theorem 3. A linear subspace $G$ of a metric linear space $(X, d)$ has the property $R_{G}(x)=\emptyset$ for every $x \in X \backslash G$ if there exists no $z \in X \backslash\{0\}$ such that $G \perp z$.

Proof. Suppose there exists some $z \in X \backslash\{\emptyset\}$ such that $G \perp z$, i.e., $g \perp z$ for every $g \in G$. Then

$$
d(g, \alpha z) \geq d(g, 0)
$$

for all $g \in G$ and for all scalars $\alpha$. This gives

$$
d(0, g) \leq d(z, g)
$$

for all $g \in G$, i.e., $0 \in R_{G}(z)$. Thus, $R_{G}(z) \neq \emptyset$ for $z \in X \mid G$, a contradiction.

Remark 2.2. In normed linear spaces, Theorem 3 and its converse were proved in [7]. It is not known whether the converse of Theorem 3 holds in metric linear spaces. 
The following theorem on existence also connects elements of best approximation and elements of best coapproximation:

Theorem 4. If $G$ is a linear subspace of a metric linear space $(X, d)$ and $x \in X \backslash G$ then

(i) $A=\left\{g_{0} \in G: g_{0} \in \cap_{g \in G P}(g)\right\} \subset R_{G}(x)$, where $\left(g_{0}, x\right)=\left\{\alpha x+(1-\alpha) g_{0}: \alpha\right.$ scalar $\}$ is the linear manifold spanned by $g_{0}$ and $x$,

(ii) for an element $g_{0} \in G$, we have $g_{0} \in R_{G}(x)$ if $G \subset P_{\left(x-g_{0}\right)}^{-1}(0) \equiv\{z \in X: 0 \in$ $\left.P_{\left(x-g_{0}\right)}(z)\right\}$.

Proof.

(i)

$g_{0} \in A \Rightarrow g_{0} \in G$ and $g_{0} \in P_{\left(g_{0}, x\right)}(g)$ for all $g \in G$

$\Rightarrow g_{0} \in G$ and $d\left(g_{0}, g\right) \leq d\left(\alpha x+\left(1-\alpha, g_{0}, g\right)\right.$ for all $g \in G$ and all scalars $\alpha$ $\Rightarrow g_{0} \in G$ and $d\left(g_{0}, g\right) \leq d(x, g)$ for all $g \in G$

i.e., $g_{0} \in R_{G}(x)$.

(ii)

$$
\begin{aligned}
G \subset P_{\left(x-g_{0}\right)}^{-1}(0) & \Rightarrow 0 \in P_{\left(x-g_{0}\right)}(g) \text { for all } g \in G \\
& \Rightarrow d(g, 0) \leq d\left(\alpha\left(x-g_{0}\right), g\right) \text { for all } g \in G
\end{aligned}
$$

Let $g^{\prime} \in G$. Take $g=g^{\prime}-g_{0}$ and $\alpha=1$. We get $d\left(g^{\prime}-g_{0}, 0\right) \leq d\left(x-g_{0}, g^{\prime}-g_{0}\right)$, i.e., $d\left(g_{0}, g^{\prime}\right) \leq d\left(x, g^{\prime}\right)$. Therefore, $g_{0} \in R_{G}(x)$.

Remark 2.3. Theorem 4 (i) clearly implies that $A=\left\{g_{0} \in G: 0 \in \cap_{g \in G} P_{\left(x-g_{0}\right)}(g-\right.$ $\left.\left.g_{0}\right)\right\} \subset R_{G}(x)$.

It was proved by Papini and Singer [13] that in normed linear spaces $R_{G}(x)=A$ in (i) and the converse part of (ii) also holds. For locally convex spaces with a family of seminorms the equality of the sets $R_{G}(x)$ and $A$ was proved by Geetha S. Rao and S. Elumalai [16].

\section{Characterizations of Coproximinal, Co-Semi-Chebyshev and Co-Cheby- shev Subspaces}

In this section, we shall characterize coproximinal, co-semi-Chebyshev, and co-Chebyshev subspaces of metric linear spaces.

It was proved in [8] that a linear subspace $G$ of a metric linear space $(X, d)$ is proximinal if and only if $X=G+P_{G}^{-1}(0)$, where $P_{G}^{-1}(0)=\left\{x \in X: 0 \in P_{G}(x)\right\}$. Analogously, 
we have the following characterization of coproximinal linear subspaces of metric linear spaces.

Theorem 5. For a linear subspace $G$ of a metric linear space $(X, d)$, the following statements are equivalent:

(a) $G$ is coproximinal.

(b) $X=G+R_{G}^{-1}(0)$.

Proof. $(a) \Rightarrow(b)$. Let $x \in X$. Since $G$ is coproximinal, there exists $g_{0} \in G$ such that $g_{0} \in R_{G}(x)$ and so by Observation 2.3 (ii), $x-g_{0} \in R_{G}^{-1}(0)$. Since $x=g_{0}+\left(x-g_{0}\right) \in$ $G+R_{G}^{-1}(0)$, we get $X \subset G+R_{G}^{-1}(0) \subset X$ and so $X=G+R_{G}^{-1}(0)$.

$(b) \Rightarrow(a)$. Let $x \in X=G+R_{G}^{-1}(0)$. Then $x=g_{0}+y, g_{0} \in G, y \in R_{G}^{-1}(0)$ and so $0 \in R_{G}(y)=R_{G}\left(x-g_{0}\right)$. Therefore, by Observation 2.3 (ii), $g_{0} \in R_{G}(y)$ implying that $G$ is coproximinal.

It was proved in [8] that a linear subspace $G$ of a metric linear space $(X, d)$ is proximinal if and only if $G$ is closed and for the canonical mapping $W_{G} ; X \rightarrow \frac{X}{G}$, we have $W_{G}\left[P_{G}^{-1}(0)\right]=\frac{X}{G}$. Analogously, we have the following characterization of coproximinal linear subspaces of metric linear spaces:

Theorem 6. For a linear subspace $G$ of a metric linear space $(X, d)$, the following statements are equivalent:

(a) $G$ is coproximinal.

(b) $G$ is closed and for the canonical mapping $W_{G}: X \rightarrow \frac{X}{G}$, we have $W_{G}\left[R_{G}^{-1}(0)\right]=\frac{X}{G}$, i.e., $W_{G}$ maps $R_{G}^{-1}(0)$ onto $\frac{X}{G}$.

Proof. $(a) \Rightarrow(b)$. Firstly, we show that $G$ is closed. Let $p \in \bar{G} \backslash G$ and $g_{0} \in R_{G}(p)$. Then there exists a sequence $\left\langle g_{n}\right\rangle$ in $G$ such that $\left\langle g_{n}\right\rangle \rightarrow p$ and $d\left(g_{0}, g\right) \leq d(p, g)$ for all $g \in G$ and so $d\left(g_{0}, g_{n}\right) \leq d\left(p, g_{n}\right.$ for all $n$. This in the limiting case implies that $\left\langle g_{n}\right\rangle \rightarrow g_{0}$ and so $p=g_{0} \in G$. Hence $G$ is closed. Now suppose $x+G \in \frac{X}{G}$ and $g_{0} \in R_{G}(x)$. Then by Observation 2.3 (ii), $x-g_{0} \in R_{G}^{-1}(0)$ and $W_{G}\left(x-g_{0}\right)=\left(x-g_{0}\right)+G=x+G$.

$(b) \Rightarrow(a)$. Let $x \in X$. Then $x+G \in \frac{X}{G}=W_{G}\left[R_{G}^{-1}(0)\right]$, i.e., $x+G=W_{G}(y)$ where $y \in R_{G}^{-1}(0)$, i.e., $x+G=y+G$ where $0 \in R_{G}(x)$, i.e., $x-y=g_{0} \in G$ and $0 \in R_{G}\left(x-g_{0}\right)$. So, by Observation 2.3 (ii), $g_{0} \in R_{G}(x)$. Hence $G$ is coproximinal.

The following characterization of Chebyshev subspace of metric linear spaces was given in [8].

For a closed linear subspace $G$ of a metric linear space $(X, d)$, the following statements are equivalent:

(i) $G$ is a Chebyshev subspace.

(ii) $X=G \oplus P_{G}^{-1}(0)$, where $\oplus$ means that the sum decomposition of each $x \in X$ is unique. 
(iii) $G$ is proximinal and $\left[P_{G}^{-1}(0)-P_{G}^{-1}(0) \cap G=\{0\}\right.$.

(iv) $G$ is proximinal and the restriction map $W_{G} \mid P_{G}^{-1}(0)$ is one to one.

Analogously, we have the following characterization of co-Chebyshev subspaces of metric linear spaces:

Theroem 7. For a closed linear subspace $G$ of a metric linear space $(X, d)$, the following statements are equivalent:

(a) $G$ is a co-Chebyshev subspace.

(b) $X=G \oplus R_{G}^{-1}(0)$, where $\oplus$ means that the sum decomposition of each $x \in X$ is unique.

(c) $G$ is coproximinal and $\left[R_{G}^{-1}(0)-R_{G}^{-1}(0)\right] \cap G=\{0\}$.

(d) $G$ is coproximinal and the restriction map $W_{G} \mid R_{G}^{-1}(0)$ is one to one.

Proof. $(a) \Rightarrow(b)$. Since $G$ is co-Chebyshev, it is coproximinal and so by Theorem 5 , $X=G+R_{G}^{-1}(0)$. Now we show that the sum decomposition of each $x \in X$ is unique. Suppose $x \in X$ and $x=g_{1}+y_{1}$ and $x=g_{2}+y_{2}$ where $g_{1}, g_{2} \in G, y_{1}, y_{2} \in R_{G}^{-1}(0)$. This gives $g_{1}-g_{2}=y_{2}-y_{1}$. Now, $y_{1} \in R_{G}^{-1}(0) \Rightarrow 0 \in R_{G}\left(y_{1}\right) \Rightarrow g_{1} \in R_{G}\left(y_{1}+g_{1}\right)$ by Observation 2.3 (iii). i.e., $g_{1} \in R_{G}(x)$. Similarly, $g_{2} \in R_{G}(x)$. Since $G$ is co-Chebyshev, $g_{1}=g_{2}$ and consequently, $y_{1}=y_{2}$. Hence, $X=G \oplus R_{G}^{-1}(0)$.

(b) $\Rightarrow(c) . X=G \oplus R_{G}^{-1}(0) \Rightarrow G$ is coproximinal by Theorem 6 . Suppose $0 \neq y \in$ $\left[R_{G}^{-1}(0)-R_{G}^{-1}(0)\right] \cap G$. Then $y=y_{1}-y_{2}, y_{1} \in R_{G}^{-1}(0), y_{2} \in R_{G}^{-1}(0), y_{1} \neq y_{2}$, So $0 \in R_{G}\left(y_{1}\right), 0 \in R_{G}\left(y_{2}\right)$. Now $y_{1}, y_{2} \in R_{G}^{-1}(0), y_{1}-y_{2} \in G \backslash\{0\}$ and $y_{1}=0+y_{1}=$ $\left(y_{1}+y_{2}\right)+y_{2}$, a contradiction to the uniqueness of the sum decomposition. Hence, $\left[R_{G}^{-1}(0)-R_{G}^{-1}(0)\right] \cap G=\{0\}$.

$(c) \Rightarrow(d)$. Suppose $W_{G} \mid R_{G}^{-1}(0)$ is not one to one, i.e., there exists $y_{1}, y_{2} \in R_{G}^{-1}(0)$, $y_{1} \neq y_{2}$ and $W_{G}\left(y_{1}\right)=W_{G}\left(y_{2}\right)$. Then $0 \neq y_{1}-y_{2} \in\left[R_{G}^{-1}(0)-R_{G}^{-1}(0)\right] \cap G$, a contradiction.

$(d) \Rightarrow(a)$. Suppose $x \in X$ has two distinct best coapproximation in $G$, say $g_{1}$ and $g_{2}$. Then by Observation 2.3 (ii), $x-g_{1}$ and $x-g_{2} \in R_{G}^{-1}(0), x-g_{1} \neq x-g_{2}$ but $W_{G}\left(x-g_{1}\right)=W_{G}\left(x-g_{2}\right)$ as $\left(x-g_{1}\right)-\left(x-g_{2}\right)=g_{2}-g_{1} \in G$ which is a contradiction.

Remark 3.1. By requiring instead of (b) that each element $x \in X$ has at most one sum decomposition and by omitting in (c) and (d) the condition of coproximinality of $G$, we obtain the following characterizations of co-semi-Chebyshev subspaces of metric linear spaces:

Theorem 8. For a closed linear subspace $G$ of a metric linear space $(X, d)$, the following statements are equivalent:

(a) $G$ is co-semi-Chebyshev subspace.

(b) Each element $x \in X$ has atmost one sum decomposition as $G+R_{G}^{-1}(0)$.

(c) $\left[R_{G}^{-1}(0)-R_{G}^{-1}(0)\right] \cap G=\{0\}$. 
(d) $W_{G} \mid R_{G}^{-1}(0)$ is one to one.

\section{The Best Coapproximation Map $\mathbb{R}_{G}$}

In this section, we shall discuss some properties of the best coapproximation map $R_{G}$ and conditions under which the mapping $R_{G}$ is upper semi-continuous or continuous.

Theorem 9. If $G$ is a subset of a metric linear space $(X, d)$ and $x \in X$, the set-valued mapping $R_{G}$ has the following properties:

(a) $D\left(R_{G}\right) \supset G$ and $R_{G}(x)=\{x\}$ for all $x \in G$.

(b) If $x \in D\left(R_{G}\right)$, then $R_{G}(x) \in D\left(R_{G}\right)$ and $R_{G}^{2}(x)=R_{G}(x)$, i.e., the mapping $R_{G}$ is idempotent on $D\left(R_{G}\right)$.

(c) If $x \in D\left(R_{G}\right)$ and $P_{G}(x) \neq \emptyset$ then $d\left(x, R_{G}^{0}(x)\right) \leq 2 d\left(x, P_{G}^{0}(x)\right), R_{G}^{0}(x) \in R^{G}(x)$ and $P_{G}^{0}(x) \in P_{G}(x)$.

(d) If $0 \in G$ then $d\left(R_{G}^{0}(x), 0\right) \leq d(x, 0)$ for all $x \in D\left(R_{G}\right)$ and $R_{G}^{0}(x) \in R_{G}(x)$. So $R_{G}$ is continuous at the origin and is a bounded mapping, in fact $R_{G}(x) \subset B(0, d(x, 0))$.

(e) If $G$ is a linear subspace and $R_{G}$ is a single-valued on $D\left(R_{G}\right)$ then for $x \in D\left(R_{G}\right)$ and $g \in G$, we have $x+g \in D\left(R_{G}\right)$ and

$$
R_{G}(x+g)=R_{G}(x)+R_{G}(g)=R_{G}(x)+g
$$

i.e., $R_{G}$ is quasi-additive.

(f) If $G$ is a linear subspace and $R_{G}^{-1}(0)$ is a closed linear subspace of $X$, then $R_{G}$ is single-valued and additive on $D\left(R_{G}\right)$.

\section{Proof.}

(a) Let $g_{0} \in G$ then $g_{0} \in R_{G}\left(g_{0}\right)$ as $d\left(g_{0}, g\right) \leq d\left(g_{0}, g\right)$ for all $g \in G$ and so $g_{0} \in$ $D\left(R_{G}\right)$. Thus, $G \subset D\left(R_{G}\right)$. Further, $g_{0} \in R_{G}\left(g_{0}\right) \Rightarrow\left\{g_{0}\right\} \subset R_{G}\left(g_{0}\right)$. Now, suppose $y \in R_{G}\left(g_{0}\right)$. Then $d(y, g) \leq d\left(g_{0}, g\right)$ for all $g \in G$ and so in particular, $d\left(y, g_{0}\right) \leq$ $d\left(g_{0}, g_{0}\right)=0$ and so $y=g_{0}$. Therefore, $R_{G}\left(g_{0}\right) \subset\left\{g_{0}\right\}$. Hence, $R_{G}(x)=\{x\}$ for all $x \in G$.

(b) Let $x \in D\left(R_{G}\right)$ then $R_{G}(x) \subset G \subset D\left(R_{G}\right)$ by Part (a). Further, $R_{G}(x) \in G \Rightarrow$ $R_{G}\left[R_{G}(x)\right]=R_{G}(x)$, i.e., $R_{G}^{2}(x)=R_{G}(x)$.

(c) $x \in D\left(R_{G}\right) \Rightarrow R_{G}(x) \neq \emptyset$. Let $R_{G}^{0}(x) \in R_{G}(x)$ and $P_{G}^{0}(x) \in P_{G}(x)$. Now, $R_{G}^{0}(x) \in$ $R_{G}(x) \Rightarrow d\left(R_{G}^{0}(x), g\right) \leq d(x, g)$ for all $g \in G \Rightarrow d\left(R_{G}^{0}(x), P_{G}^{0}(x)\right) \leq d\left(x, P_{G}^{0}(x)\right)$ as $P_{G}^{0}(x) \in G$.

Consider,

$$
\begin{aligned}
d\left(x, R_{G}^{0}(x)\right) & \leq d\left(x, P_{G}^{0}(x)\right)+d\left(P_{G}^{0}(x), R_{G}^{0}(x)\right) \\
& \leq 2 d\left(x, P_{G}^{0}(x)\right)
\end{aligned}
$$


(d) $x \in D\left(R_{G}\right) \Rightarrow R_{G}(x) \neq \emptyset$. Let $R_{G}^{0}(x) \in R_{G}(x)$. Then

$$
d\left(R_{G}^{0}(x), g\right) \leq d(x, g)
$$

for all $g \in G$ implies

$$
d\left(R_{G}^{0}(x), 0\right)=\leq d(x, 0) .
$$

The continuity of $R_{G}$ at origin is now immediate. Also, $R_{G}(x) \subset B(0, d(x, 0))$ and so $R_{G}$ is a bounded mapping.

(e) Suppose $R_{G}$ is single valued on $D\left(R_{G}\right), x \in D\left(R_{G}\right)$ and $g \in G$.

$$
\begin{aligned}
x \in D\left(R_{G}\right) & \Rightarrow d\left(R_{G}(x), g^{\prime}\right) \leq d\left(x, g^{\prime}\right) \text { for all } g^{\prime} \in G \\
& \Rightarrow d\left(R_{G}(x)+g, g^{\prime}+g\right) \leq d\left(x+g, g^{\prime}+g\right) \text { for all } g^{\prime} \in G \\
& \Rightarrow d\left(R_{G}(x)+g, g^{*}\right) \leq d\left(x+g, g^{*}\right) \text { for all } g^{*} \in G \\
& \Rightarrow R_{G}(x)+g \in R_{G}(x+g) .
\end{aligned}
$$

Consequently, $x+g \in D\left(R_{G}\right)$ and since $R_{G}$ is single valued, $R_{G}(x+g)=R_{G}(x)+g=$ $R_{G}(x)+R_{G}(g)$, by (a). Thus, $R_{G}$ is quasiadditive.

(f) Let $x \in D\left(R_{G}\right)$ and $g_{1}, g_{2} \in R_{G}(x)$. Then by Observation 2.3 (ii), $x-g_{1}, x-g_{2} \in$ $R_{G}^{-1}(0)$. Since $R_{G}^{-1}(0)$ is a linear subspace, $\left(x-g_{1}\right)-\left(x-g_{2}\right) \in R_{G}^{-1}(0)$, i.e., $g_{2}-g_{1} \in R_{G}^{-1}(0) \cap G=\{0\}$ and so $g_{2}=g_{1}$, i.e., $R_{G}$ is single valued on $D\left(R_{G}\right)$.

Now we show that $R_{G}$ is additive on $D\left(R_{G}\right)$. Let $x, t \in D\left(R_{G}\right)$ and $R_{G}(x)=g_{1}$, $R_{G}(y)=g_{2}$. Then by Observation 2.3 (ii), $x-g_{1}, y-g_{2} \in R_{G}^{-1}(0)$. Since $R_{G}^{-1}(0)$ is a linear subspace, $\left(x-g_{1}\right)+\left(y-g_{2}\right) \in R_{G}^{-1}(0)$. So, $0=R_{G}\left(x+y-g_{1}-g_{2}\right)$. Consider,

$$
\begin{aligned}
R_{G}(x+y)-\left(g_{1}+g_{2}\right) & =R_{G}(x+y)-R_{G}\left(g_{1}+g_{2}\right) \\
& =R_{G}\left(x+y-g_{1}-g_{2}\right), \text { by }(\mathrm{e}) \\
& =0 \\
& =R_{G}(x)-g_{1}+R_{G}(x)-g_{2} .
\end{aligned}
$$

This gives $R_{G}(x+y)=R_{G}(x)+R_{G}(y)$, i.e., $R_{G}$ is additive on $D\left(R_{G}\right)$.

Remark 4.1. In normed linear spaces, Property (a) was observed in [3], Properties (b) and (e) in [13], and Property (f) in [7]. Properties (a), (b) and (e) were proved in locally convex spaces in [11] and [16]. Properties (a) to (e) are also true for metric projections in metric linear spaces (see Pantelidis [12]).

We may recall that a mapping $T: X \rightarrow 2^{Y}$ where $X$ and $Y$ are metric spaces and $2^{Y}$ denotes the collection of all subsets of $Y$, is said to be upper semi-continuous if the set

$$
\{x \in X: T(x) \cap N \neq \emptyset\}
$$

is closed for every clsoed $N \subset Y$. 
Now, we discuss some conditions under which the mapping $R_{G}$ is upper semi-continuous or continuous.

Theorem 10. If $G$ is a closed linear subspace of a metric linear space $(X, d)$ then $R_{G}$ is upper semi-continuous on $D\left(R_{G}\right)$ if and only if for each closed subset $N$ of $G$, $N+R_{G}^{-1}(0)$ is closed.

Proof. Suppose $R_{G}$ is upper semi-continuous and $N$ is a closed subset of $G$. Let $x$ be a limit point of $N+R_{G}^{-1}(0)$. Then there exists a sequence $\left\langle x_{n}\right\rangle$ in $N+R_{G}^{-1}(0)$ such that $x_{n} \rightarrow x$. Suppose $x_{n}=g_{n}+y_{n}$ where $g_{n} \in N$ and $y_{n} \in R_{G}^{-1}(0)$. Since $y_{n}=x_{n}-g_{n} \in R_{G}^{-1}(0)$, by Observation 2.3 (ii) $g_{n} \in R_{G}\left(x_{n}\right) \cap N$. The upper semicontinuity of $R_{G}$ implies $R_{G}(x) \cap N \neq \emptyset$ and so there exists some $g \in R_{G}(x) \cap N$. This gives $x-g \in R_{G}^{-1}(0)$, i.e., $x \in N+R_{G}^{-1}(0)$ and so $N+R_{G}^{-1}(0)$ is closed.

Conversely, suppose $N+R_{G}^{-1}(0)$ is closed for each closed subset $N$ of $G$. Suppose $R_{G}$ is not upper semi-continuous on $D\left(R_{G}\right)$. Then there exists an $x \in D\left(R_{G}\right)$ and a sequence $\left\langle x_{n}\right\rangle$ in $D\left(R_{G}\right)$ such that $x_{n} \rightarrow x, R_{G}\left(x_{n}\right) \cap N \neq \emptyset$ but $R_{G}(x) \cap N=\emptyset$. So, there exists $g_{0} \in R_{G}(x)$ such that $g_{0} \notin N$ and so $x \notin N+R_{G}^{-1}(0)$, contradicting that $N+R_{G}^{-1}(0)$ is closed. Hence $R_{G}$ is upper semi-continuous.

Remark 4.2. For normed linear spaces, Theorem 10 was proved in [18]. Some more results on the upper semicontinuity of the mapping $R_{G}$ given in normed linear spaces in [18], were proved in metric spaces in [10].

Next theorem proves the upper semi-continuity of $R_{G}$ when $R_{G}^{-1}(0)$ is boundedly compact (i.e., when every bounded sequence in $R_{G}^{-1}(0)$ has a subsequence converging to an element of $X$ ). For normed linear spaces this result was stated in [18].

Theorem 11 . If $G$ is a closed linear subspace of a metric linear space $(X, d)$ such that $R_{G}^{-1}(0)$ is boundedly compact, then $R_{G}(x)$ is compact and $R_{G}$ is upper semi-continuous on $D\left(R_{G}\right)$.

Proof. Let $\left\langle g_{n}\right\rangle$ be an arbitrary sequence in $R_{G}(x)$, i.e.,

$$
d\left(g_{n}, g\right) \leq d(x, g)
$$

for all $g \in G$. Then $\left\langle x-g_{n}\right\rangle$ is a bounded sequence in $R_{G}^{-1}(0)$ and so, it has a subsequence $\left\langle x-g_{n_{i}}\right\rangle \rightarrow x-g_{0} \in R_{G}^{-1}(0)$ as $R_{G}^{-1}(0)$ is also closed by Observation 2.3 (i). Consequently, $\left\langle g_{n}\right\rangle$ has a subsequence $\left\langle g_{n_{i}}\right\rangle \rightarrow g_{o} \in R_{G}(x)$ and hence $R_{G}(x)$ is compact.

Now suppose $N$ is a closed subset of $G$ and

$$
B=\left\{x \in D\left(R_{G}\right): R_{G}(x) \cap N \neq \emptyset\right\} .
$$

To show $B$ is closed, let $x$ be a limit point of $B$. Then there exists a sequence $\left\langle x_{n}\right\rangle$ in $B$ such that $x_{n} \rightarrow x$. Now $x_{n} \in B \Rightarrow$ there exists $g_{n} \in R_{G}\left(x_{n}\right) \cap N, n=1,2, \cdots$ So

$$
d\left(g_{n}, g\right) \leq d\left(x_{n}, g\right)
$$


for all $g \in G$. This gives $x_{n}-g_{n} \in R_{G}^{-1}(0)$ and is a bounded sequence as $\left\langle x_{n}\right\rangle$ and $\left\langle g_{n}\right\rangle$ are both bounded. Since $R_{G}^{-1}(0)$ is boundedly compact, there is a subsequence $\left\langle x_{n_{i}}-g_{n_{i}}\right\rangle \rightarrow x-g_{0} \in R_{G}^{-1}(0)$ as $R_{G}^{-1}(0)$ is also closed by Observation 2.3 (i). This gives $g_{0} \in R_{G}(x) \cap N$, i.e., $x \in B$. Hence $R_{G}$ is upper semi-continuous.

Remark 4.3. In case $R_{G}$ is single-valued (this is so if $G$ is co-semi-Chebyshev), Theorem 10 and 11 give the continuity of $R_{G}$ on $D\left(R_{G}\right)$ and on $X$ if $G$ is also coproximinal.

Remark 4.4. If $R_{S, G}(x)$ is the set of all those elements of $G$ which belong to $R_{G}(x)$ strongly (we say that ' $g_{0} \in R_{G}(x)$ strongly' if $x \notin G$ and there exists an $r(0<r \leq 1$ ) such that $d\left(g_{0}, g\right)+r d\left(g_{0}, x\right) \leq d(x, g)$ for all $\left.g \in G\right)$, the mapping $R_{S, G}: x \rightarrow R_{S, G}(x)$ defined on $D\left(R_{S, G}\right)=\left\{x \in X: R_{S, G}(x) \neq \emptyset\right\}$, is called 'strong best coapproximation map.' For linear subspace $G$ of normed linear spaces, Theorem 10,11, and some other results have been proved for $R_{S, G}$ in [17].

\section{Acknowledgements}

The authors are thankful to the referee fo valuable comments leading to an improvement of the paper. The first author is also thankful to U. G. C. (India) for financial support.

\section{References}

[1] H. Berens and U. Westphal, On the best coapproximation in a Hilbert space, in 'Quantitative Approximation' (R. A. DeVore and K. Scherer, Eds., Academic Press, New York (1980), 7-10.

[2] G. Birkhoff, Orthogonality in linear metric spaces, Duke Math J. 1(1935), 169-172.

[3] C. Franchetti and M. Furi, Some characteristic properties of real Hilbert spaces, Rev. Roum. Math. Pures Appl. 17(1972), 1045-1048.

[4] L. Hetzelt, On suns and cosuns in finite dimensional normed real vector spaces, Acta Math. Hung. 45(1985), 53-68.

[5] J. A. Johnson, Banach spaces of Lipschitz functions and vector-valued, Lipschitz functions, Trans. Amer. Math. Soc. 148(1970), 147-169.

[6] T. D. Narang, On certain characterizations of best approximation in metric linear spaces, Pure Appl. Mathematika Sciences, 4(1976), 121-124.

[7] T. D. Narang, On best coapproximation, Ranchi Univ. Math. J. 17(1986), 49-56.

[8] T. D. Narang, Best approximation in metric linear spaces, Math. Today, 5(1987), 21-28.

[9] T. D. Narang, On best coapproximation in normed linear spaces, Rocky Mountain J. Math., 22(1991), 265-287.

[10] T. D. Narang, Best coapproximation in metric spaces, Publ. Inst. Math. 51(1992), 71-76.

[11] T. D. Narang and S. P. Singh, Best coapproximation in locally convex spaces, Tamkang J. Math 28(1997), 1-5.

[12] G. Pantelidis, Approximationstheorie für metrische lineare Räume, Math. Annal. 184 (1969), 30-48. 
[13] P. L. Papini and I. Singer, Best approximation in normed linear spaces, Math. Mh. 88(1979), 27-44.

[14] Geetha S. Rao, Best coapproximation in normed linear spaces: Approximation Theory, Vol. V, Eds. Chui, Schumaker and Ward, Academic Press, New York (1986), 535-538.

[15] Geetha S. Rao and K. R. Chandrasekran, Characterisations of elements of best coapproximation in normed linear spaces, Pure Appl. Mathematika Scineces 26(1987), 139-147.

[16] Geetha S. Rao and S. Elumalai, Approximation and strong approximation in locally convex spaces, Pure Appl. Mathematika Sciences 19(1984), 13-26.

[17] Geetha S. Rao and S. Elumalai, Semi-continuity properties of the strong best coapproximation operator, Indian J. Pure Appl. Math. 16(1985), 257-270.

[18] Geetha S. Rao and S. Muthukumar, Semi-continuity properties of the coapproximation operator, Math. Today, 5(1987), 37-48.

[19] I. Singer, Best approximation in normed linear spaces by elements of linear subspaces, Springer-Verlag, New York, 1970.

[20] U. Westphal, Cosuns in $\ell^{p}(n), 1 \leq p<\infty$, J. Approx. Theory, 54(1988), 287-305.

Department of Mathematics, Guru Nanak Dev University, Amritsar-143005, India.

Department of Mathematics and Statistics, Memorial University of Newfoundland, St. John's, NF, A1C 5S7, Canada. 